\title{
DISTRIBUIÇÃO, FRACIONAMENTO E MOBILIDADE DE ELEMENTOS TRAÇO EM SEDIMENTOS SUPERFICIAIS
}

\section{Janice Cardoso Pereira}

Centro Federal de Educação Tecnológica de Minas Gerais, Av. Amazonas, 5253, 30480-000 Belo Horizonte - MG, Brasil

Aline Kelly Guimarães-Silva e Hermínio Arias Nalini Júnior

Departamento de Geologia, Escola de Minas, Universidade Federal de Ouro Preto, 35400-000 Ouro Preto - MG, Brasil

Érica Pacheco-Silva e Jorge Carvalho de Lena*

Departamento de Química, Instituto de Ciências Exatas e Biológicas, Universidade Federal de Ouro Preto, 35400-000

Ouro Preto - MG, Brasil

Recebido em 25/8/06; aceito em 20/12/06; publicado na web em 30/7/07

\begin{abstract}
DISTRIBUTION, FRACTIONATION AND MOBILITY OF TRACE ELEMENTS IN STREAM SEDIMENTS. The gold and iron mining activities in the Velhas River yield considerable amounts of suspended solids as well as metals to the hydrological system of the region. The risks were assessed by sequential extraction procedures. They were carried out in sediment samples, aiming to evaluate the presence of $\mathrm{Co}, \mathrm{Cr}, \mathrm{Cu}, \mathrm{Ni}, \mathrm{Zn}$ and As. Although being at high levels in some parts of the river (e.g. As $527 \mathrm{mg} \mathrm{g}^{-1}$ and $\mathrm{Cr} 475 \mathrm{mg} \mathrm{g}^{-1}$ ) they are associated with the residual fraction. Mobility factors were also calculated and show that these species are enriched along the river, e.g. for As 0.02 at the spring and 0.33 at the high course of the river.
\end{abstract}

Keywords: sediments; trace elements; fractionation.

\section{INTRODUÇÃO}

Os minerais presentes na crosta terrestre são as fontes primárias de metais para o ambiente. Os elementos químicos liberados não são formados e nem destruídos, mas redistribuídos por diferentes tipos de mecanismos. Conseqüentemente, estes elementos não são encontrados uniformemente disseminados nas camadas mais superficiais da crosta e nem mesmo entre os seus vários compartimentos, como rochas, solos e sedimentos marinhos e continentais ${ }^{1}$.

Os sedimentos são sólidos suspensos ou depositados que atuam como o principal componente de uma matriz, que foi ou é susceptível ao transporte pelas águas ${ }^{2}$. São partes essencial, integral e dinâmica das bacias hidrográficas, incluindo estuários e zonas costeiras. Sua origem está associada ao intemperismo e erosão de rochas e solos das porções mais altas da área de drenagem ${ }^{3}$. Os sedimentos apresentam valor ecológico, social e econômico. Do ponto de vista ecológico é importante ressaltar que o sedimento é fonte de recursos energéticos para o compartimento biótico e é o componente-chave para o estudo da integridade ecológica destes ambientes ${ }^{2,4}$.

Em ambientes aquáticos, os íons metálicos e os metalóides podem estar distribuídos entre as fases solúveis em água, coloidal, material em suspensão e uma grande proporção dessas espécies é incorporada aos sedimentos de fundo ${ }^{5}$. De modo geral, os sedimentos têm uma alta capacidade de reter espécies químicas orgânicas e inorgânicas e menos de $1 \%$ das substâncias que atingem um sistema aquático são dissolvidas em água e, conseqüentemente, mais de $99 \%$ são estocadas no compartimento sedimentar ${ }^{6}$. Portanto, a análise química de sedimentos fornece uma eficiente ferramenta para o gerenciamento da qualidade hídrica ${ }^{3}$. No entanto, a legislação brasileira, que regulamenta a qualidade desses recursos hídricos, não prevê tais procedimentos (resolução CONAMA № 357, de 17/03/05).

*e-mail: jorge.delena@iceb.ufop.br
Geralmente, a variação da concentração de elementos químicos em sedimentos pode ser caracterizada em função de fatores como a geologia da região, o uso e ocupação da área do entorno, o regime hidrológico, o tipo de vegetação, a presença de mineralizações, dentre outros ${ }^{7}$. A avaliação da concentração total de metais e metalóides em sedimentos de ecossistemas aquáticos naturais não é suficiente para predizer a sua capacidade de mobilização $0^{8-12}$. Assim, a concentração total tem uma importância sob o ponto de vista estocástico, mas fornece poucos dados sob o ponto de vista ambiental.

Metais traço em sedimentos podem existir em diferentes formas químicas. Quando estes compartimentos não sofrem ação antrópica, os metais traço estão principalmente ligados aos silicatos e aos minerais primários e, portanto, com baixo grau de mobilidade. Já aqueles elementos químicos incorporados a partir de atividades antrópicas apresentam maior mobilidade e estão ligados a outras fases do sedimento, como carbonatos, óxidos, hidróxidos e sulfetos. Diferentes variáveis como a gênese dos sedimentos e os processos que controlam o transporte e a redistribuição dos elementos, como adsorção, dessorção, precipitação, solubilização e floculação, determinam o comportamento desses ambientes ${ }^{13}$.

As técnicas de extração seqüencial são comumente usadas para o fracionamento de metais em solos, sedimentos e outras matrizes ambientais e buscam avaliar o potencial de mobilidade e disponibilidade destes metais ${ }^{14}$. Diferentes métodos para a avaliação das formas de associação destes elementos em matrizes sólidas estão descritos na literatura científica ${ }^{15-17}$. Os métodos mais amplamente empregados são aqueles baseados no procedimento de extração em várias etapas, nas quais diferentes reagentes são consecutivamente empregados para extrair metais das frações operacionalmente definidas, de acordo com as recomendações da IUPAC ${ }^{18}$.

Vários autores ${ }^{14,19,20}$ têm discutido problemas de falta de seletividade, readsorção e o longo tempo demandado para a aplicação da técnica de extração seqüencial, tornando esta metodologia sujeita a uma série de contaminações que têm como conseqüência 
a baixa repetibilidade dos resultados. Nirel e Morel $^{21}$ criticaram severamente a falta de validação dos procedimentos analíticos de extração seqüencial aplicados para amostras ambientais complexas. No entanto, estes esquemas de extração em única ou em múltiplas etapas têm sido amplamente adotados ${ }^{22}$ e adaptados para várias matrizes em diferentes estudos ambientais, condizentes com um expressivo número de publicações recentes sobre este tema ${ }^{23-30}$.

Neste trabalho foi utilizado um sistema de extração seqüencial em três etapas sugerido pelo "Community Bureau of Reference", agora "Standards, Measurements and Testing Programme", que foi desenvolvido com o objetivo de harmonizar os diferentes procedimentos descritos na literatura. No procedimento $\mathrm{BCR}^{31}$, as frações operacionalmente definidas como as solúveis em ácido, redutível e oxidável, são obtidas através de ataques químicos sucessivos, utilizando na primeira etapa uma solução de ácido acético $0,11 \mathrm{~mol} \mathrm{~L}^{-1}$, na segunda cloridrato de hidroxilamina, $0,5 \mathrm{~mol} \mathrm{~L}^{-1}$, na terceira uma solução de peróxido de hidrogênio $8,8 \mathrm{~mol} \mathrm{~L}^{-1}$ e posteriormente acetato de amônio 1,0 mol L'-1 e finalmente o resíduo da etapa 3 é digerido com água régia e identificado como fração residual. Para complementar estes dados e para efeito de comparação, uma extração simples ${ }^{30,32}$ é obtida com $\mathrm{HCl} 0,1 \mathrm{~mol} \mathrm{~L}^{-1}$, à temperatura ambiente e a "pseudo-total" é obtida com água régia, a quente.

Este artigo teve como objetivos: estudar a distribuição de elementos traço em sedimentos de superfície do alto da bacia do rio das Velhas; avaliar a mobilidade desses elementos com base nos estudos de fracionamento, de acordo com o esquema de extração seqüencial em três etapas; comparar os dados de extração seqüencial com extração em fase única utilizando $\mathrm{HCl} 0,1 \mathrm{~mol} \mathrm{~L}^{-1}$ e digestão com água régia e, finalmente, correlacionar dados geoquímicos e mineralógicos para gerar informações sobre a origem, disponibilidade e mobilidade de metais traço em sedimentos do alto do rio das Velhas.

\section{PARTE EXPERIMENTAL}

\section{Área de estudo}

O estado de Minas Gerais representa um dos maiores potenciais minerários do país. Por conseqüência, os problemas ambientais advindos desta atividade extrativo-mineral na região são extremamente relevantes. Dentro desse contexto, no Quadrilátero Ferrífero (QF), onde se encontra uma expressiva parte das atividades de mineração do estado, os problemas ambientais acentuam-se ainda mais, exercendo um forte impacto sobre os recursos hídricos. O QF é uma região montanhosa que apresenta concentrações econômicas de ferro, ouro, manganês, alumínio e topázio ${ }^{33}$.

A porção mais alta da bacia do rio das Velhas, região de nascentes, localizada a sudeste do QF, apresenta intensa atividade de mineração e conseqüente geração de efluentes com elevados teores de sólidos em suspensão e diferentes espécies metálicas. Esta área é também de grande importância socioeconômica porque fornece água para toda a região metropolitana de Belo Horizonte, em diversas modalidades como para consumo humano, uso agropecuário e industrial ${ }^{34}$.

$\mathrm{O}$ alto do rio das Velhas corta as três grandes unidades litoestratigráficas que compõem o Quadrilátero Ferrífero: Embasamento Cristalino - inclui um complexo gnáissico e magmatítico; Supergrupo Rio das Velhas - composto por "greenstone belt" (basalto e komatiito), riolito e rocha sedimentar intercalada e Supergrupo Minas - cujas unidades basais são compostas por conglomerados e arenitos gradando para pelitos marinhos, que foram recobertos por uma seqüência de sedimentos químicos do Grupo Itabira representados pela formação ferrífera bandada ${ }^{35}$.
Os 20 segmentos fluviais investigados estão apresentados na Figura 1 , onde 8 pontos $(00 ; 01 ; 02 ; 04 ; 07 ; 16 ; 18$ e 21$)$ estão localizados no rio das Velhas e os demais $(03 ; 05 ; 06 ; 08 ; 09 ; 10$; $11 ; 12 ; 13 ; 14 ; 15$ e 20$)$ em seus tributários mais expressivos, distribuídos nas margens direita e esquerda. Dezenove sítios amostrais foram selecionados a partir da rede operada no projeto Águas de Minas e de uma rede mais adensada e dirigida para o alto curso do rio das Velhas, montada no âmbito do Programa Nacional de Meio Ambiente - PNMA-II. Ambos os programas de monitoramento são coordenados pelo IGAM (Instituto Mineiro de Gestão das Águas) e abrangem um total de 36 estações de amostragem ${ }^{36}$. Além destes, um ponto mais a montante, identificado como 00 , foi incluído na rede e é considerado como área de controle, uma vez que sofre menor pressão ambiental. Foram estudados $\mathrm{Co}, \mathrm{Cr}, \mathrm{Cu}, \mathrm{Ni}, \mathrm{Zn}$ e As, devido à abundância destes elementos nos litotipos da região.

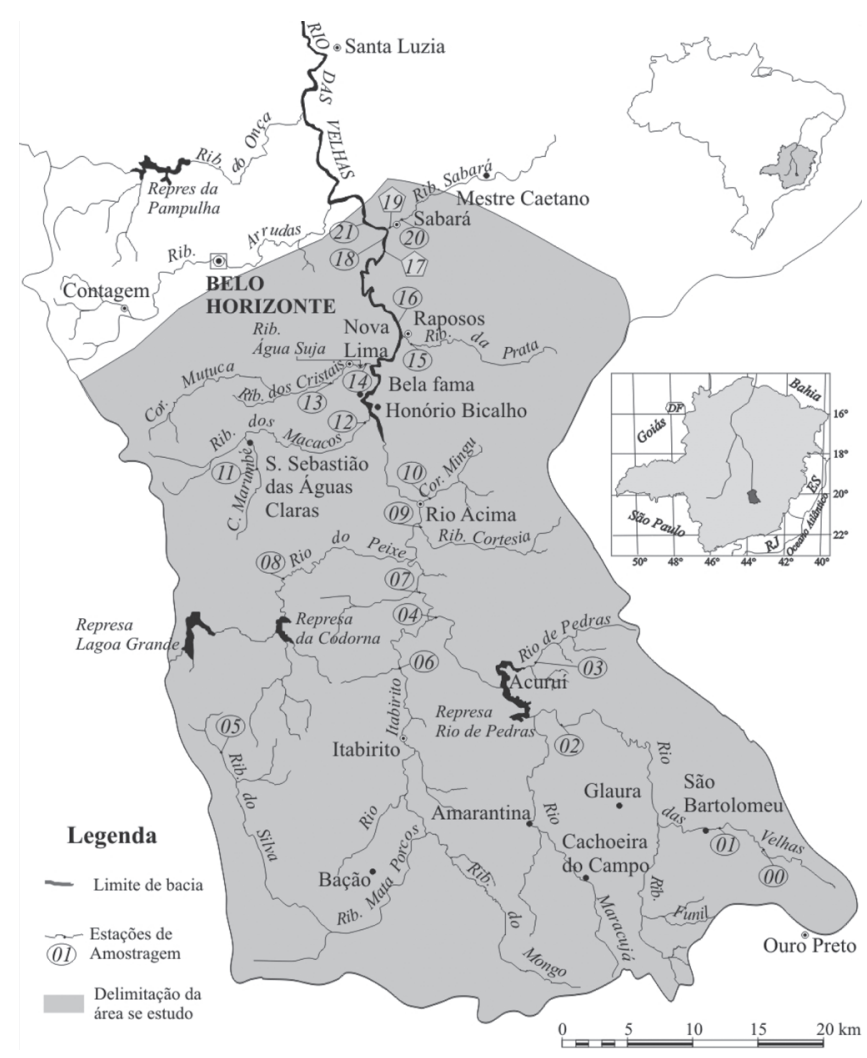

Figura 1. Mapa da área de estudo

\section{Amostragem e preparo das amostras}

Para amostragem de sedimentos foram escolhidas áreas de remanso, onde a menor energia do sistema fluvial favorece o depósito de material mais fino e, portanto, de maior interesse para avaliação ambiental do comportamento dos elementos químicos ${ }^{3}$. No período de junho a agosto de 2002, cerca de $2 \mathrm{~kg}$ de amostras de sedimentos superficiais compostas foram coletadas em cada segmento fluvial, de acordo com as recomendações do USGS ${ }^{37}$, utilizando-se um amostrador de polietileno manufaturado no laboratório e acondicionadas em sacos plásticos. No laboratório, as amostras foram transferidas para recipientes plásticos e colocadas em uma capela com exaustão forçada, onde foi adaptado um sistema de aquecimento com lâmpadas de infravermelho e a temperatura controlada a $40 \pm 5^{\circ} \mathrm{C}$. Após este procedimento, as amostras foram desagregadas, quarteadas, onde $2 / 4$ foram reservados e outros $2 / 4$ foram fracionados por peneiramento, sendo a fração mais fina $(<63 \mu \mathrm{m})$ tomada para estes experimentos. 


\section{Vidrarias e reagentes}

Todas as vidrarias e frascos utilizados nos procedimentos foram imersos em solução de $\mathrm{HNO}_{3} 1 \mathrm{~mol} \mathrm{~L}^{-1}$ por $24 \mathrm{~h}$ e abundantemente enxaguados em água deionizada. Todos os reagentes utilizados foram de grau de pureza analítica, ou superior, e as soluções foram preparadas utilizando água ultrapura, obtida a partir do sistema de purificação da marca Milli-Q, Millipore ${ }^{\circledR}$. As curvas analíticas foram preparadas a partir de soluções-padrão para ICP, das marcas J.T. Baker e Merck, de $1.000 \mathrm{mg} \mathrm{L}^{-1}$ para todos os elementos, exceto para ferro que o padrão foi de $10.000 \mathrm{mg} \mathrm{L}^{-1}$.

\section{Digestão "pseudo-total” com água régia}

Para a digestão "pseudo-total" foram adicionados $10 \mathrm{~mL}$ de uma mistura de $\mathrm{HCl} / \mathrm{HNO}_{3}$, na proporção de $3: 1$, a uma massa de 1 $\mathrm{g} \pm 0,1 \mathrm{mg}$ de amostra de sedimento, que permaneceu em repouso no béquer tampado com vidro de relógio por $16 \mathrm{~h}$, à temperatura ambiente. Após este período, as amostras foram aquecidas por $2 \mathrm{~h}$ $\left(80{ }^{\circ} \mathrm{C}\right)$, resfriadas e, em seguida, filtradas, transferidas e avolumadas para balão volumétrico de $50 \mathrm{~mL}$. Após a homogeneização, as soluções foram acondicionadas em frascos tipo PET, lacradas, identificadas e mantidas a $4{ }^{\circ} \mathrm{C}$ até a leitura.

\section{Extração em fase única - fração ambientalmente disponível}

A fração potencialmente disponível em sedimentos é definida, em termos operacionais, como a fração extraída por um ataque ácido moderado ${ }^{38}$ e neste trabalho é denominada ambientalmente disponível. Massas de 0,5 g $\pm 0,1 \mathrm{mg}$ de amostras de sedimentos foram pesadas em frascos de polipropileno para centrífuga e $20 \mathrm{~mL}$ de solução de ácido clorídrico $0,1 \mathrm{~mol} \mathrm{~L}^{-1}$ foram adicionados. Os frascos tampados foram colocados em um agitador rotativo $360^{\circ}$, marca Heidoph Instruments, Reax 2, à temperatura ambiente, por $16 \mathrm{~h}$ e, em seguida, foram centrifugados durante $30 \mathrm{~min}$, a 3000 rpm e o sobrenadante separado, acondicionado em frascos tipo PET e mantidos a $4{ }^{\circ} \mathrm{C}$ até a leitura.

\section{Extração seqüencial - frações operacionalmente definidas}

A metodologia adotada para o fracionamento por extração seqüencial, em três fases mais a fase residual, foi desenvolvida pelo "Standard, Measurements and Testing Program" - BCR ${ }^{31}$. Uma sinopse deste protocolo está descrita na Tabela 1. O material de referência, BCR-701, foi utilizado para assegurar a qualidade da aplicação de tais procedimentos e brancos analíticos foram avaliados em todas as fases da extração. A Tabela 2 apresenta os resulta- dos das taxas de recuperação calculados para cada etapa do procedimento de extração seqüencial para cinco dos seis elementos químicos certificados, sendo eles o $\mathrm{Cd}, \mathrm{Cr}, \mathrm{Cu}, \mathrm{Ni}$ e $\mathrm{Zn}$. Os valores das taxas de recuperação variaram entre 80 e $136 \%$, considerando o valor médio sugerido pela literatura.

Este esquema de extração seqüencial em três etapas, devido à alta reprodutibilidade dos resultados e a aplicabilidade em diferentes matrizes de sedimentos, tem sido usado como método de referência em muitos laboratórios. A certificação deste material de referência (BCR 701) mostrou que os sedimentos secos permanecem estáveis por um longo período de tempo à temperatura ambiente, além de permitir um controle de qualidade e validação das técnicas analíticas empregadas ${ }^{14,39}$.

\section{Difratômetro de raios-X}

Para a caracterização mineralógica utilizou-se um equipamento, marca Shimadzu, modelo XRD 6000, equipado com tubo de cobalto $(\mathrm{Co})$ e filtro de ferro $(\mathrm{Fe})$. As varreduras foram feitas entre 7 e $70^{\circ}(2 \theta)$ com velocidade de $2^{\circ} / \mathrm{min}$.

\section{Espectrofotômetro de emissão óptica}

Todas as análises de metais foram realizadas em um equipamento ICPOES radial, da marca Spectro, modelo Ciros CCD. Várias curvas analíticas, com diferentes faixas de concentrações dos elementos, foram preparadas com as soluções extratoras empregadas em cada etapa do procedimento de extração seqüiencial e em fase única, visando a correção de matriz ${ }^{40}$. Um ajuste na curva analítica, utilizada para digestão com água régia, foi feito adicionando ácido clorídrico em quantidade equivalente à percentagem de ácido residual na amostra após esta digestão. As linhas analíticas selecionadas foram sempre as de maior sensibilidade para os elementos traço e, dependendo da matriz, estas foram substituídas por outras de melhor sinal analítico e livre de interferência. Todo o procedimento foi realizado em duplicata.

\section{RESULTADOS E DISCUSSÃO}

\section{Distribuição espacial de metais e arsênio}

A digestão das amostras foi feita a partir de um ataque com água régia e, portanto representa a concentração "pseudo-total" destes elementos. Esta digestão disponibiliza, além dos metais mais lábeis, aqueles de menor mobilidade que estão associados aos óxidos cristalinos de ferro e manganês, matéria orgânica, sulfetos e alguns silicatos mais alterados ${ }^{41}$. Estes resultados po-

Tabela 1. Protocolo analítico $\mathrm{BCR}^{31}$, adaptado para $1 \mathrm{~g}$ de amostra

\begin{tabular}{|c|c|c|c|c|}
\hline Fração & Reagentes & Volume (mL) & Temp. $\left({ }^{\circ} \mathrm{C}\right)$ & Tempo de extração \\
\hline Fração 3 (Oxidável) & $8,8 \mathrm{~mol} \mathrm{~L}^{-1}$ de $\mathrm{H}_{2} \mathrm{O}_{2}$ & 10 & $\begin{array}{l}22 \pm 5 \\
85 \pm 2\end{array}$ & $\begin{array}{c}\text { Digestão por } 1 \mathrm{~h} \text { com agitação ocasional } \\
\text { Digestão por } 1 \mathrm{~h}\end{array}$ \\
\hline Fração 4 (Residual) & Água Régia $\mathrm{HCl} / \mathrm{HNO}_{3}(3: 1)$ & 10 & $\begin{array}{l}22 \pm 5 \\
85 \pm 2\end{array}$ & $\begin{array}{c}\text { Repouso por } 16 \mathrm{~h} \\
\text { Aquecimento por } 2 \mathrm{~h}, \\
\text { com agitação ocasional }\end{array}$ \\
\hline
\end{tabular}


Tabela 2. Taxa de recuperação para o material de referência BCR 701

\begin{tabular}{cccccc}
\hline & Fração 1 & Fração 2 & Fração 3 & Fração 4 & $\begin{array}{c}\text { Digestão } \\
\text { "pseudototal" }\end{array}$ \\
\hline & & & $\%$ & & \\
$\mathrm{As}$ & $\mathrm{NC}$ & $\mathrm{NC}$ & $\mathrm{NC}$ & $\mathrm{NC}$ & $\mathrm{NC}$ \\
$\mathrm{Co}$ & $\mathrm{NC}$ & $\mathrm{NC}$ & $\mathrm{NC}$ & $\mathrm{NC}$ & $\mathrm{NC}$ \\
$\mathrm{Cr}$ & 112 & 91 & 108 & 115 & 96 \\
$\mathrm{Cu}$ & 102 & 87 & 112 & 136 & 93 \\
$\mathrm{Ni}$ & 92 & $\mathrm{ND}$ & 80 & 96 & 103 \\
$\mathrm{Zn}$ & 102 & 103 & 82 & 113 & 90 \\
\hline
\end{tabular}

$\mathrm{NC}$ - valores não certificados $\mathrm{ND}$ - valores não determinados

dem estar subestimados se considerada a contribuição de metais associados à rede cristalina dos minerais à base de silicatos e, portanto, resistentes a este ataque químico. Esta digestão, no entanto, é bem aceita para estudos do comportamento de elementos químicos em matrizes ambientais e seus resultados são usados como valores de referência para avaliar o grau de mobilidade de metais e metalóides associados a outras frações operacionalmente definidas do sedimento ${ }^{31}$. A distribuição dos elementos analisados, de montante para jusante, é apresentada na Figura 2. O ponto AV-14 (córrego do Cardoso) apresenta valores de As, Co, Ni, Cu e $\mathrm{Zn}$ que se destacam do conjunto, sugerindo um alto grau de impactação naquele ponto. $\mathrm{O} \mathrm{Cr}$ apresenta altos valores na nascente (AV-00 e AV-01) e no ponto AV-11, mas, como veremos, este elemento está ligado à fase residual naqueles sítios, não significando necessariamente impactação.

\section{Ocorrência e abundância de metais e arsênio}

A avaliação da qualidade dos sedimentos muitas vezes é feita através da comparação dos resultados obtidos com diferentes VGQS (Valores Guias de Qualidade para Sedimentos), disponíveis na literatura $^{42,43}$. A maioria destes valores foi estabelecida através de uma abordagem empírica baseada em informações de extensos bancos de dados sobre níveis de contaminação, valores de referência natural ("baseline"), ensaios ecotoxicológicos e, portanto, devem ser usados e interpretados com cautela. É importante ressaltar que muitos destes VGQS são definidos em função da concentração total dos elementos no sedimento, desconsiderando as fases mais lábeis, ou seja, aquelas de maior importância na interpretação de dados dentro de uma abordagem ambiental.

Na legislação brasileira não existem critérios estabelecidos para avaliar a qualidade dos sedimentos, no entanto a CETESB - Companhia de Tecnologia de Saneamento Ambiental do Estado de São Paulo - adota os valores canadenses - TEL/PEL ${ }^{42}$ - para classificar e regulamentar a disposição de sedimentos dragados. Visando compreender a ocorrência e abundância de metais e arsênio na região em estudo, dois valores guias distintos foram comparados com as concentrações de metais nas amostras de sedimentos do alto da bacia do rio das Velhas.

Os valores utilizados foram TEL/PEL ${ }^{42}$, sendo TEL - nível limiar de efeitos ("Threshold Effect Concentration") e o PEL - nível provável de efeitos ("Probable Effect Level") e ERL/ERM ${ }^{43}$, sendo ERL intervalo de efeito baixo ("Effect Range-Low") e ERM - intervalo de efeito médio ("Effect Range-Mediam"). Estes VGQS diferem em valores nominais propostos para cada metal, mas todos têm como objetivo a proteção da vida em ambientes aquáticos. Geralmente as faixas destes valores propostos são divididas em três partes: abaixo do valor mínimo sugerido, onde raramente um efeito adverso é esperado; entre o valor mínimo e o valor máximo, quando provavelmente um efeito adverso é esperado e, superior ao valor máximo sugerido, quando freqüentemente se espera um efeito adverso.

A Tabela 3 mostra os VGQS TEL/PEL e ERL/ERM para As, $\mathrm{Cd}, \mathrm{Cr}, \mathrm{Cu}, \mathrm{Ni}$ e $\mathrm{Zn}$ e o número de sítios amostrais (n) que se encontram dentro de cada faixa proposta para os valores guias adotados. Considerando somente estes critérios para avaliar a qualidade dos sedimentos, observa-se que dos 20 segmentos fluviais estudados todos extrapolam valores de PEL para Cd, 18 para Ni e 16 para Cr. Já para valores de ERL/ERM que são menos restritivos, em 18 dos sítios amostrais os valores excedem o limite superior para $\mathrm{Cd}$, para Ni são 13 ocorrências e, no caso do $\mathrm{Cr}$, 11 valores

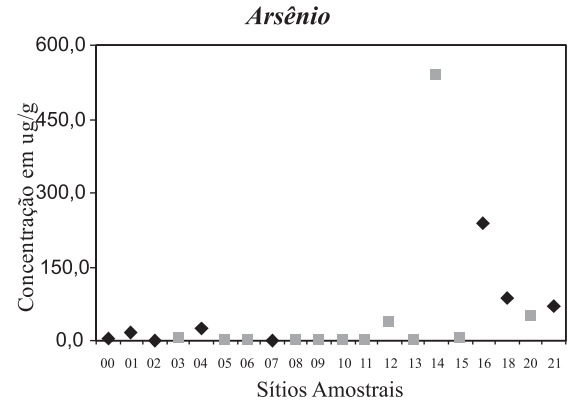

Cobre

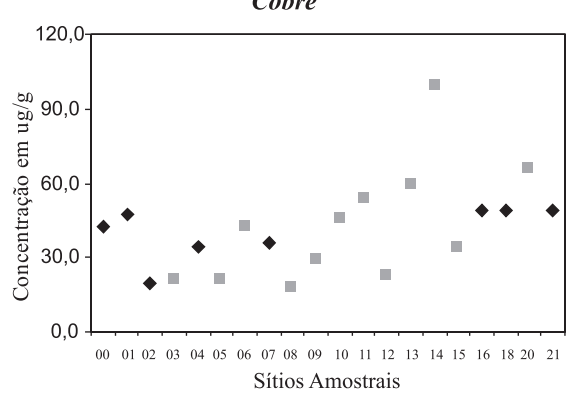

Cobalto

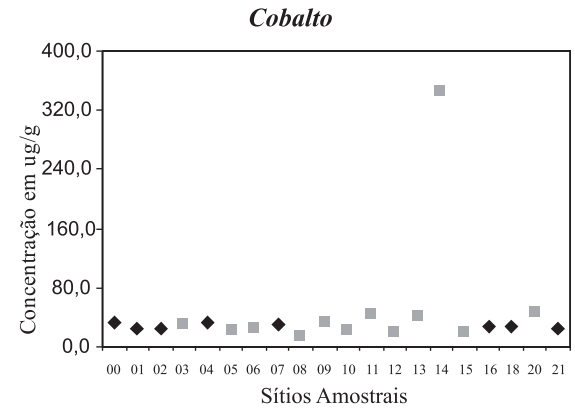

Níquel

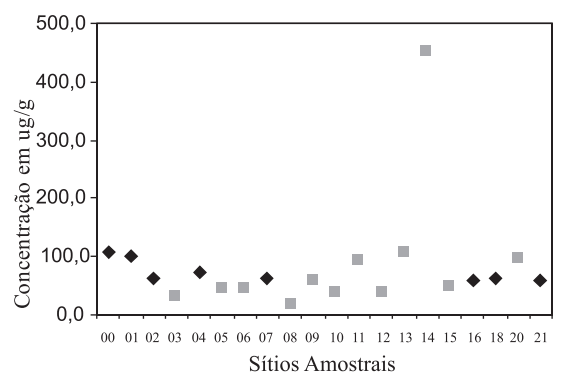

Cromo
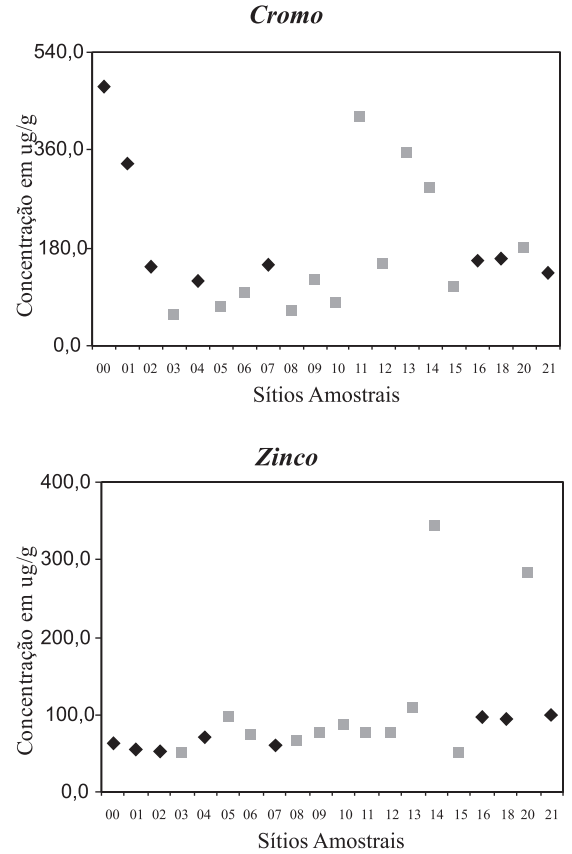

Figura 2. Distribuição espacial de As, Co, Cr, Cu e Zn ao longo dos vinte segmentos do alto curso da bacia do Rio das Velhas. (•) Alto rio das Velhas, (回) Tributários do Alto Velhas 
Tabela 3. Valores Guias de Qualidade de Sedimentos (em $\left.\mu \mathrm{g} \cdot \mathrm{g}^{-1}\right)$ e o número de amostras (n) dentro das faixas de cada VGQS.

\begin{tabular}{|c|c|c|c|c|c|c|c|c|c|c|}
\hline & $\mathrm{TEL}^{42}$ & $\mathrm{PEL}^{42}$ & $\begin{array}{c}<\mathrm{TEL} \\
\mathrm{n}\end{array}$ & $\begin{array}{c}>\mathrm{TEL} \\
<\mathrm{PEL} \\
\mathrm{n}\end{array}$ & $\begin{array}{c}>\text { PEL } \\
n\end{array}$ & $\mathrm{ERL}^{43}$ & $\mathrm{ERM}^{43}$ & $\begin{array}{c}<\text { ERL } \\
n\end{array}$ & $\begin{array}{c}>\text { ERL } \\
<\text { ERM } \\
n\end{array}$ & $>$ ERM \\
\hline As & 5,9 & 17 & 12 & 0 & 8 & 33 & 85 & 16 & 1 & 3 \\
\hline $\mathrm{Cr}$ & 37,3 & 90,0 & 0 & 4 & 16 & 80 & 145 & 4 & 5 & 11 \\
\hline $\mathrm{Cu}$ & 35,7 & 197 & 8 & 12 & 0 & 70 & 390 & 19 & 1 & 0 \\
\hline $\mathrm{Ni}$ & 18,0 & 36,0 & 0 & 2 & 18 & 30 & 50 & 1 & 6 & 13 \\
\hline $\mathrm{Zn}$ & 123 & 315 & 18 & 1 & 1 & 120 & 270 & 18 & 0 & 2 \\
\hline
\end{tabular}

estão acima dos sugeridos para ERM.

Para o arsênio, apesar de somente oito amostras extrapolarem os limites superiores para os dois critérios, no sítio 14 de comportamento anômalo, a concentração é cerca de 7 vezes maior que o limite superior para ERL/ERM e 32 vezes maior que os limites sugerido por TEL/PEL. Já para o cobre e zinco quase todos os segmentos fluviais apresentam concentrações destes elementos abaixo do nível inferior, ou entre o limite inferior e o superior. Estes VGQS adotados não fornecem dados para o cobalto e, portanto, este elemento não foi avaliado.

\section{Extração seqüencial - frações operacionalmente definidas}

Para o estudo do fracionamento das espécies estudadas, seis segmentos fluviais foram selecionados, priorizando dois pontos mais a montante (00 e 01), dois tributários de maior expressão (14 e 20) e dois pontos mais a jusante (16 e 21). A Figura 3 apresenta os resultados deste experimento, com valores expressos em percentuais extraídos em cada fração para os seis elementos avaliados.

De modo geral, os elementos químicos nos seis sedimentos fracionados têm um comportamento similar, sendo a fração residual a mais significativa e a fração oxidável a menos expressiva. A concentração de carbono orgânico oxidável é pouco significativa sendo que o valor máximo não ultrapassou a $10,1 \mathrm{mg} \mathrm{g}^{-1} \mathrm{e}$, considerando-se que os sedimentos são oriundos de ambientes essencialmente lóticos, os resultados são condizentes com as características físico-químicas. Por outro lado, apesar dos sedimentos analisados serem enriquecidos em ferro, as espécies analisadas não estão preferencialmente associados à fração redutível.

A contribuição da fração de maior interesse ambiental, mais lábil, não é expressiva para a maioria dos elementos, com exceção do cobalto para todas as amostras e o cobre e zinco para os sítios 14, 20 e 21. De todos os elementos estudados, o cobalto é o que se apresenta homogeneamente distribuído entre as frações trocável, redutível e residual. $\mathrm{O}$ arsênio e o crômio apresentam comportamento similar e encontram-se preferencialmente associados à fração residual e, portanto, menos móvel em todos os 6 sítios analisados.

De montante para jusante, seguindo os 6 segmentos fluviais investigados dentro da bacia do rio das Velhas, observa-se uma tendência dos elementos apresentarem maior mobilidade, ou seja,
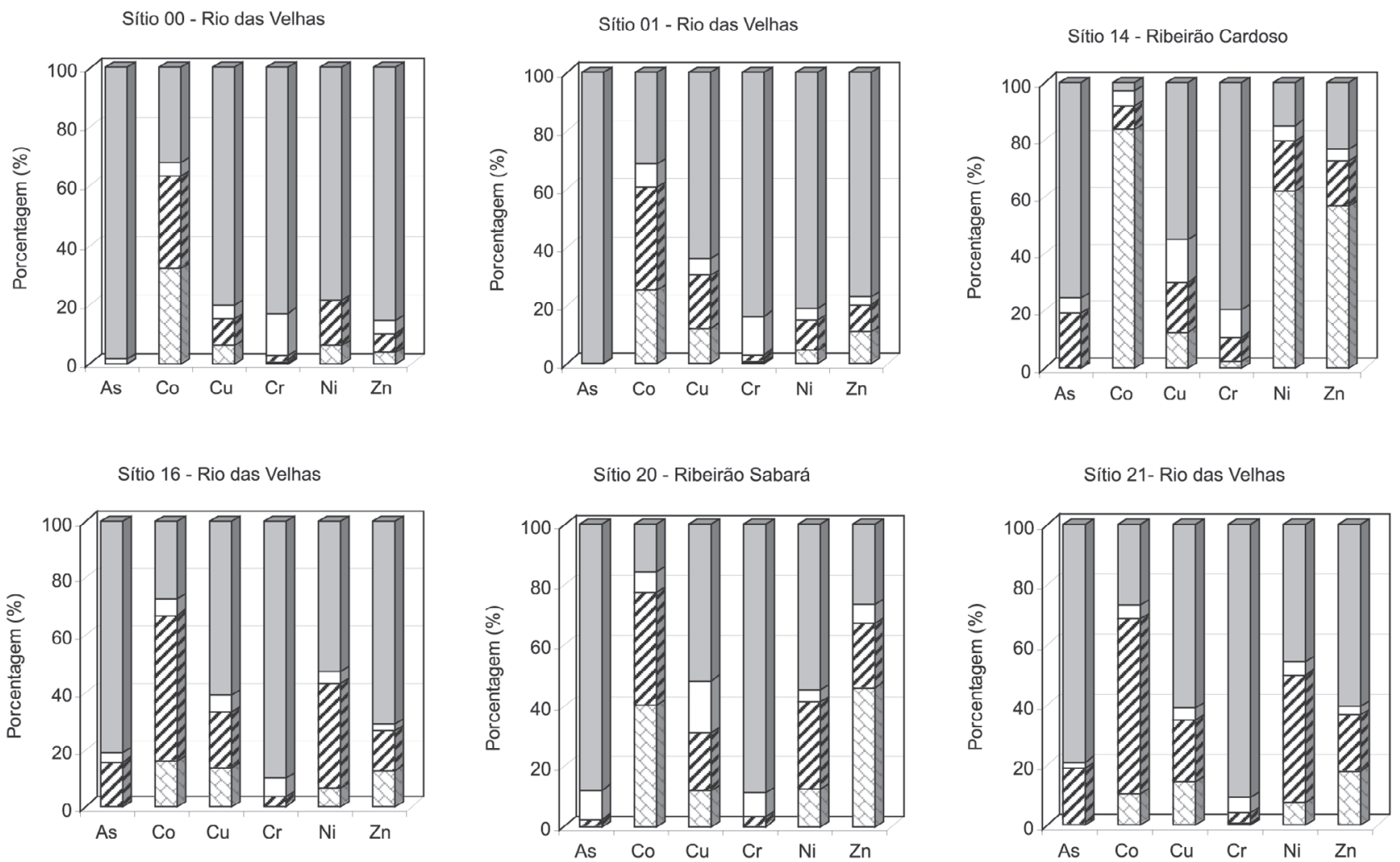

Figura 3. Fracionamento de As, Co, Cr, Cu e Zn em seis segmentos fluviais do alto curso da bacia do Rio das Velhas. (四) Trocável, (ש) Redutível, ( $\square$ ) Oxidável, (口) Residual 
a distribuição dos elementos químicos nas frações mais lábeis, principalmente a trocável e redutível, aumenta e isto pode ser mais bem observado para $\mathrm{Ni}, \mathrm{Zn}$ e As.

Para o sítio 14, os resultados do fracionamento mostram um maior potencial de mobilidade dos elementos, principalmente para Co, Ni e Zn, já para o arsênio a fração redutível é a mais significativa. Estes dados acentuam a preocupação com a concentração de metais e As neste segmento fluvial.

\section{Fração ambientalmente disponível}

O conceito de disponibilidade de metais é muito utilizado e uma variedade de soluções pode ser empregada para estimar esta porção mais lábil em sedimentos e outras matrizes de importância ambiental ${ }^{44}$. As amostras dos 6 segmentos fluviais, pré-selecionados para o fracionamento em três etapas, foram submetidas a uma extração em fase única com $\mathrm{HCl} 0,1 \mathrm{~mol} \mathrm{~L}^{-1}$. As percentagens extraídas para cada elemento foram comparadas com as percentagens obtidas na soma das três etapas do processo de extração seqüencial e os resultados estão apresentados na Tabela 4. De modo geral, para o $\mathrm{As}, \mathrm{Cu}$ e $\mathrm{Zn}$, a extração em fase única é similar à soma das frações em três etapas e para o $\mathrm{Co}, \mathrm{Cr}$ e $\mathrm{Ni}$ os extratores usados no fracionamento em três etapas foram mais eficientes. Para o sítio 14 , fortemente impactado, a extração com $\mathrm{HCl} 0,1 \mathrm{~mol} \mathrm{~L}^{-1}$ apresenta eficiência semelhante à extração em três etapas, para todos os elementos, com exceção para o $\mathrm{Co}$ e $\mathrm{Cr}$, onde a soma das três frações mostrou maior eficiência.

\section{Implicações ambientais}

A avaliação da integridade ecológica de sistemas fluviais é complexa e envolve o conhecimento de processos físicos, quími- cos e biológicos. No entanto, dados físico-químicos podem se tornar ferramentas úteis para se avaliar os riscos ambientais e estimar possíveis danos causados aos organismos bentônicos. A Tabela 5 apresenta os fatores de mobilidade para cada elemento $\left(\mathrm{M}_{\mathrm{f}}^{\mathrm{i}}\right)$ e o fator de mobilidade global $\left(\mathrm{M}_{\mathrm{f}}\right)$, que foram adaptados de Fernandes ${ }^{45}$. $\mathrm{O}$ fator de mobilidade individual para cada amostra foi calculado a partir da soma das concentrações de cada elemento extraído nas três fases do processo de extração seqüencial, dividido pela concentração deste elemento na fração residual e o fator de mobilidade global foi obtido a partir da soma dos valores da mobilidade individual.

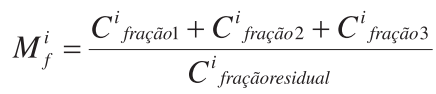

$$
\begin{aligned}
& M_{f}=\sum M_{f}^{i}
\end{aligned}
$$

Fernandes ${ }^{45}$ utilizou o mesmo fator, mas denominando fator de contaminação, para avaliar o impacto ambiental causado por cinco elementos ( $\mathrm{Cd}, \mathrm{Pb}, \mathrm{Cu}, \mathrm{Cr}$ e $\mathrm{Zn}$ ) na laguna de Jacarepaguá, RJ. O maior coeficiente individual calculado para este corpo d'água foi de 5,62 para o cobre. Já Da Silva et al. ${ }^{46}$ aplicaram estes fatores para avaliar as condições ambientais do sistema fluvial Tietê-Pinheiros, SP e o fator de mobilidade global para seis elementos $(\mathrm{Cu}, \mathrm{Pb}, \mathrm{Cd}, \mathrm{Zn}, \mathrm{Ni}$ e $\mathrm{Cr}$ ) variou de 2,9 \pm 0,7 para uma área considerada de controle (Barra Bonita) até $48 \pm 8$ para a represa Billings, reconhecidamente poluída por metais e diferentes substâncias orgânicas.

Os fatores de mobilidade global calculados para o alto curso do rio das Velhas confirmam um enriquecimento de metais e As, nas frações mais disponíveis e ao longo do fluxo da bacia, ou seja, estes índices aumentam no sentido sítio $00<01<16<21$. Os tributários 14 e 20, notoriamente mais impactados, apresentam os

Tabela 4. Comparação entre percentagens extraídas na soma das três etapas do processo de extração seqüencial com a extração em uma única fase $\left(\mathrm{HCl} 0,1 \mathrm{~mol} \mathrm{~L}^{-1}\right)$

\begin{tabular}{|c|c|c|c|c|c|c|c|}
\hline \multirow[t]{2}{*}{ Sítios Amostrais } & \multirow[t]{2}{*}{ Extrações } & As & Co & $\mathrm{Cu}$ & $\mathrm{Cr}$ & $\mathrm{Ni}$ & $\mathrm{Zn}$ \\
\hline & & \multicolumn{6}{|c|}{ \% extraída } \\
\hline \multirow[t]{2}{*}{00} & Seqüencial & 1,3 & 68,0 & 20,0 & 18,0 & 11,0 & 14,7 \\
\hline & Fase única & $\sim 0$ & 31,3 & 18,2 & 0,7 & 6,1 & 8,4 \\
\hline \multirow[t]{2}{*}{01} & Seqüencial & $\sim 0$ & 69,0 & 37,0 & 17,0 & 20,0 & 23,0 \\
\hline & Fase única & $\sim 0$ & 31,8 & 31,8 & 1,0 & 4,9 & 17,6 \\
\hline \multirow[t]{2}{*}{14} & Seqüencial & 24,0 & 96,7 & 46,0 & 21,0 & 85,0 & 77,0 \\
\hline & Fase única & 19,9 & 30,5 & 30,5 & 6,6 & 83,7 & 79,1 \\
\hline \multirow[t]{2}{*}{16} & Seqüencial & 19,0 & 73,0 & 39,0 & 39,0 & 48,0 & 29,0 \\
\hline & Fase única & 5,4 & 37,5 & 37,5 & 37,5 & 12,0 & 22,8 \\
\hline \multirow[t]{2}{*}{20} & Seqüencial & 12,0 & 53,0 & 38,0 & 38,0 & 45,0 & 73,0 \\
\hline & Fase única & 16,7 & 43,2 & 43,2 & 43,2 & 11,5 & 65,0 \\
\hline \multirow[t]{2}{*}{21} & Seqüencial & 11,0 & 73,0 & 39,9 & 39,9 & 55,0 & 40,0 \\
\hline & Fase única & 4,8 & 35,5 & 35,5 & 35,5 & 13,6 & 29,0 \\
\hline
\end{tabular}

Tabela 5. Fator de mobilidade individual ${ }^{45}\left(\mathrm{M}_{\mathrm{f}}^{\mathrm{i}}\right)$ e fator de mobilidade global ${ }^{46}\left(\mathbf{M}_{\mathrm{f}}\right)$

\begin{tabular}{lccccccc}
\hline SítiosAmostrais & As & Co & $\begin{array}{c}\mathrm{Cu} \\
\mathrm{M}_{\mathrm{f}}^{\mathrm{i}}\end{array}$ & $\mathrm{Cr}$ & $\mathrm{Ni}$ & $\mathrm{Zn}$ & $\mathrm{M}_{\mathrm{f}}=\Sigma \mathrm{M}_{\mathrm{f}}^{\mathrm{i}}$ \\
\hline 00 & 0,02 & 2,12 & 0,24 & 0,20 & 0,27 & 0,17 & 3,02 \\
01 & $<0,01$ & 2,20 & 0,56 & 0,19 & 0,23 & 0,30 \\
14 & 0,33 & 31,25 & 0,82 & 0,26 & 5,74 & 3,25 & 41,65 \\
16 & 0,23 & 2,66 & 0,64 & 0,11 & 0,90 & 0,40 & 4,94 \\
20 & 0,14 & 5,40 & 0,94 & 0,13 & 0,83 & 2,78 \\
21 & 0,26 & 2,70 & 0,64 & 0,10 & 1,19 & 0,65 & 5,54 \\
\hline
\end{tabular}


maiores índices de mobilidade, sendo que no ribeirão Cardoso (sítio 14) este fator chega a 41,65 e pode ser comparado com aquele determinado para a represa Billings ${ }^{46}$. O maior fator de mobilidade individual calculado para os seis segmentos estudados foi 31,25 para o cobalto e é aproximadamente seis vezes superior ao obtido para cobre na laguna de Jacarepaguá ${ }^{47}$.

\section{CONCLUSÃO}

Os sedimentos superficiais do alto da bacia do rio das Velhas apresentam elevadas concentrações de crômio nos sítios mais a montante (sítios 00 e 01), anomalias para As, Co, Cr, Cu, Ni e Zn em amostras do ribeirão Cardoso (sítio 14), além de um enriquecimento de $\mathrm{As}, \mathrm{Cu}$ e $\mathrm{Zn}$ para os pontos mais a jusante.

Os VGQS aplicados (Tabela 3) classificam alguns segmentos fluviais como impactados, principalmente para $\mathrm{As}, \mathrm{Cd}, \mathrm{Cr}$, e Ni, podendo, segundo estes critérios, causar efeitos adversos à comunidade bentônica. No entanto, estudos de fracionamento de alguns metais e As mostram que grande parte destes elementos está associada à fração residual (Figura 3), com exceção do cobalto que se encontra homogeneamente distribuído entre todas as frações.

$\mathrm{O}$ fator de mobilidade aplicado mostra que a distribuição espacial do grau de mobilidade global dos elementos químicos segue a tendência $00<01<16<21<20<14$, sendo 20 e 14 tributários de grande expressão em volume de água e de intensa intervenção antrópica, como mineração e urbanização. $\mathrm{O}$ fator de mobilidade individual sugere uma menor mobilidade para As e $\mathrm{Cr}$ e maior para $\mathrm{Co}$, influenciando diretamente na disponibilidade destes elementos.

A comparação das percentagens extraídas usando processos de extração em três etapas e em fase única mostra a eficiência do procedimento mais simplificado para avaliação do potencial de disponibilidade para a maioria dos elementos químicos avaliados.

As técnicas e os dados discutidos neste trabalho, no contexto das complexas questões ambientais, podem ser tornar ferramentas úteis no desenvolvimento de projetos que visem uma avaliação integrada para o gerenciamento da qualidade da bacia do alto do rio das Velhas. No entanto, a otimização de protocolos analíticos de extração seqüencial e/ou em fase única se faz necessária, considerando que estes sedimentos são particularmente enriquecidos em óxidos de ferro.

\section{AGRADECIMENTOS}

À FAPEMIG - projeto CRA 925/01, à CAPES - Probral 162/ 03 e ao CNPq pelo suporte financeiro e pela bolsa PIBIC para a quarta autora.

\section{REFERÊNCIAS}

1. Smith, K. S.; Huck, H. L. O. Em The Environmental Geochemistry of Mineral Deposits Part A, Plumlee, G. S.; Logsdon, M.J., eds.; Society of Economic Geologists, Reviews in Economic Geology, 1999, cap. 2.

2. http://www.sednet.org, acessada em Junho 2005.

3. Förstner, U.; Trends Anal. Chem. 2004, 23, 217.

4. Moreira, R. C. A.; Boaventura, G. R.; Quim. Nova 2003, 26, 812.

5. Filgueiras, A. V.; Lavilla, I.; Bendicho, C.; Sci. Total Environ. 2004, 330, 115.

6. Förstner, U. Em Biogedynamics of Pollutants in Soils and Sediments Salomons, W.; Stigliani, W. M., eds.; Springer: Berlim, 1995, cap. 11.
7. Tarvainen, T.; Kallio, E.; Appl. Geochem. 2002, 17, 975.

8. Barreto, S. R. G.; Nozaki, J.; Oliveira, E.; Nascimento Filho, V. F.; Aragão, P. H. A.; Scarminio, I. S.; Barreto, W. J.; Talanta 2004, 64, 345

9. Fytianos, K.; Lourantou, A.; Environ. Int. 2004, 30, 11.

10. Sutherland, R. A.; Tack, F. M. G.; Adv. Environ. Res. 2003, 37, 50.

11. Fangueiro, D.; Bermond, A.; Santos, E.; Carapuça, H.; Duarte, A.; Talanta 2005, 66, 844 .

12. Morillo, J.; Usero, J.; Gracia, I.; Environ. Int. 2002, 28, 263.

13. Sahuquillo, A.; López-Sanches, J. F.; Rauret, G.; Ure, A. M.; Muntau, H.; Quevauviller, Ph. Em Methodologies for Soil and Sediment Fractionation Studies; Quevauviller, Ph., ed.; RSC: Cambridge, 2002, cap. 2.

14. Gleyzes, C.; Tellier, S.; Astruc, M.; Trends Anal. Chem. 2002, 21, 451.

15. Tessier, A.; Campbell, P. G. C.; Bisson, M.; Anal. Chem. 1979, 51, 844

16. Salomons, W.; Förstner, U.; Environ. Technol. Lett. 1980, 1, 506.

17. Quevauviller, Ph.; Trends Anal. Chem. 1998, 17, 289.

18. Templeton, D. M.; Ariese, F.; Cornelis, R.; Danielson, L.G.; Muntau, H.; van Leeuwen, H. P.; Lobínski, R.; Pure Appl. Chem. 2000, 72, 1453.

19. Hlavay, J.; Prohaska, T.; Weisz, M; Wenzel, W. W.; Stingeder G. J.; Pure Appl. Chem. 2004, 76, 415.

20. Egreja-Filho, F. B.; Tese de Doutorado, Universidade Federal de Viçosa, Brasil, 2000.

21. Nirel, P. M. V.; Morel, F. M. M.; Water Res. 1990, 24, 1055.

22. Ure, A. M.; Davidson, C. M.; Chemical Speciation in the Environment, $2^{\text {nd }}$ ed., Blackwell Scientific Publications: Oxford, 2002.

23. Canepari, S.; Cardarelli, E.; Ghighi, S.; Scimonelli, L.; Talanta 2005, 66, 1122.

24. Pazos-Capeáns, P.; Barciela-Alonso, M. C.; Barmejo-Barrera, A.; BarmejoBarrera, P.; Talanta 2005, 65, 678.

25. Basílio, M. S.; Friese, K.; Lena, J. C.; Nalini Jr, H. A.; Roeser, H. M. P.; Quim. Nova 2005, 28, 822 .

26. Guevara-Riba, A.; Sahuquillo, A.; Rubio, R.; Rauret, G.; Sci. Total Environ. 2004, 321, 241.

27. Gismera, M. J.; Lacal, J.; Silva, P.; Garcia, R.; Sevilla, M. T.; Procopio, J. R.; Environ. Pollut. 2004, 127, 175.

28. Morillo, J.; Usero, J.; Gracia, I.; Chemosphere 2004, 55, 431.

29. Jain, C. K.; Water Res. 2004, 38, 569.

30. Snape, I.; Scouller, R. C.; Stark, S. C.; Stark, J.; Riddle, M. J.; Gore, D. B.; Chemosphere 2004, 57, 491.

31. Rauret, G.; López-Sánches, J. F.; Lück, D.; Yli-Halla, M.; Muntau, H.; Quevauviller, Ph.; The certification of the extractable contents (mass fractions) of $\mathrm{Cd}, \mathrm{Cr}, \mathrm{Cu}, \mathrm{Ni}, \mathrm{Pb}$ and $\mathrm{Zn}$ in freshwater sediment following sequential extraction procedure - BCR 701, BCR information reference material, Brussels, Belgium, 2001.

32. Mozeto, A. A.; Silvério, P. F.; De Paula, F. C. F.; Bevilacqua, J. E.; Patella, E.; Jardim, W. F. Em Ecovision World Monograph Series; Munawar, M., ed.; 2003, p. 221.

33. Uhlein, A.; Oliveira, H. A.; Ciência Hoje 2000, 27, 160.

34. http://cbhrv.org.br, acessada em Julho 2005.

35. Alkmin, F. F.; Marshak, S.; Precambrian Res. 1998, 90, 29

36. http://www.igam.gov.mg.br, acessada em Julho 2005.

37. Shelton, L. R.; Capel, P. D.; Guidelines for collecting and processing samples of stream bed sediments for analysis of trace elements and organic contaminants for the National Water-Quality Assessment Program, USGS, 1994, Open-File Report 94-458.

38. Fiszmann, M.; Pfeiffer, W. C.; Lacerda, L. D.; Environ. Technol. Lett. 1984, $5,567$.

39. Quevauviller, Ph.; Methodologies for Soil in Sediment Fractionation Studies, RSC: Cambridge, 2002, cap. 1.

40. Grotti, M.; Ianni, C.; Frache, R.; Talanta 2002, 57, 1053.

41. Ryan, P. C.; Wall, A. J.; Hiller, S.; Clark, L.; Chem. Geol. 2002, 184, 337.

42. Smith, S. L.; MacDonald, D. D.; Keenleyside, K. A.; Ingersoll, C. G.; Field, J.; J. Great Lakes Res. 1996, 22, 624.

43. Long, E. R.; Morgan, L. G.; NOAA Technical Memorandum NOS OMA 52, National Oceanic and Atmospheric Administration: Seattle, 1991.

44. Yin, Y.; Impellitteri, C. A.; You, S-J.; Allen, H. E.; Sci. Total Environ. 2002, 287, 107.

45. Fernandes, H. M.; Environ. Pollut. 1997, 97, 317

46. Da Silva, I. S.; Abate, G.; Lichtig, J.; Masini, J. C.; Appl. Geochem. 2002, $17,105$.

47. De Paula, F. C. F.; Mozeto, A. A.; Appl. Geochem. 2001, 16, 1139. 